\title{
Pressurized Intraperitoneal Aerosol Chemotherapy Enhanced by Electrostatic Precipitation (ePIPAC) for Patients with Peritoneal Metastases
}

\author{
Abdelkader Taibi ${ }^{1,2}$ (D), Hugo Teixeira Farinha ${ }^{3}$, Sylvaine Durand Fontanier ${ }^{1,2}$, Zaid Sayedalamin ${ }^{4}$, \\ Martin Hübner ${ }^{3}$, and Olivia Sgarbura ${ }^{4}$ \\ ${ }^{1}$ Digestive Surgery Department, Visceral Surgery Department, Dupuytren Limoges University Hospital, Limoges, France; \\ ${ }^{2}$ CNRS, XLIM, UMR 7252, University Limoges, Limoges, France; ${ }^{3}$ Department of Visceral Surgery, Lausanne University \\ Hospital (CHUV), University of Lausanne (UNIL), Lausanne, Switzerland; ${ }^{4}$ Surgical Oncology Department, Montpellier \\ Cancer Institute (ICM), University of Montpellier, Montpellier, France
}

\begin{abstract}
Background. Pressurized intraperitoneal aerosol chemotherapy (PIPAC) is a new mode of intraperitoneal chemotherapy administration that can potentially be improved by the addition of electrostatic precipitation (ePIPAC). This study aimed to describe the procedural details of ePIPAC and to analyze its safety for patients with nonresectable peritoneal metastasis as well as their tolerance and response to this treatment.
\end{abstract}

Methods. This retrospective cohort study included consecutive patients treated with ePIPAC in three centers from April 2019 to April 2020. The toxicities of each patient were assessed using the Common Terminology Criteria for Adverse Events (CTCAE). Complications were documented according to the Clavien classification. Quality of life (QoL) was assessed using EORTC-QLQ-C30, and the peritoneal regression grading score (PRGS) was used to grade histologic responses. Further surrogates for responses were the Peritoneal Cancer Index (PCI), ascites, and symptoms.

Results. Overall, 69 patients received 147 ePIPACs with oxaliplatin $(n=34)$ or cisplatin/doxorubicin $(n=35)$ mainly for colorectal $(n=25)$, ovarian $(n=14)$, and gastric $(n=13)$ primary cancers. Systemic chemotherapy was

(C) Society of Surgical Oncology 2020

First Received: 8 July 2020

Accepted: 17 October 2020;

Published Online: 20 November 2020

A. Taibi

e-mail: abdelkader.taibi@hotmail.Fr used in the treatment of 54 patients (76\%). The median electrostatic therapy time was 12 min (range 6-30 min). The overall and major CTCAE toxicity rates were respectively $24.6 \%$ and $15.9 \%$. The postoperative complications rate according to Clavien classification was $4.7 \%$. The responses of 22 patients who had three or more ePIPAC treatments were evaluated as follows: PCI (16 vs 14 ; $p=0.4$ ), ascites ( $320 \mathrm{vs} 98 \mathrm{ml} ; p=0.1)$, and PRGS (2.23 vs $1.73 ; p=0.15$ ). The complete (PRGS1) and major (PRGS2) histologic responses at the third ePIPAC were respectively $38.5 \%$ and $53.8 \%$. Overall QoL was stable during the first ePIPACs.

Conclusion. Repetitive ePIPACs were safe and well tolerated for patients with unresectable peritoneal metastasis.

Pressurized intraperitoneal aerosol chemotherapy (PIPAC) was described recently as an innovative intraperitoneal (IP) drug delivery method in several experimental and clinical studies. ${ }^{1-3}$ This method was conceived to overcome the pharmacologic limitations related to the conventional IP administration such as poor drug distribution within the abdominal cavity. This innovatory concept seems to enhance the penetration of IP chemotherapy into peritoneal metastases (PMs) by taking advantage of the physical properties of gas and pressure.

A few studies have suggested that responses to PIPAC can be evaluated based on histologic response resulting from the systematic use of the peritoneal regression grade score (PRGS). ${ }^{4,5}$ Moreover, a recent systematic review confirmed that the potential benefits are associated with a limited incidence of severe adverse events (Common 
Terminology Criteria for Adverse Events [CTCAE] > 2). ${ }^{6}$ The original technique nebulizes the cytotoxic products in the peritoneal cavity and maintains the steady state for $30 \mathrm{~min}$.

To decrease the time of this surgical procedure and to improve spatial heterogeneity, some authors have suggested that adding electrostatic loading of the aerosolized particles as an adjunct to PIPAC should further enhance the pharmacologic properties of PIPAC. ${ }^{7-9}$ The addition of electrostatic precipitation was studied by the pioneer team in Tübingen. The findings suggested an enhancement of tissue drug penetration, with only 6 min waiting time after the administration, corresponding to exposure time. Nevertheless, few preclinical and clinical data are available in the literature about electrostatic precipitation PIPAC (ePIPAC).

This multicentric study aimed to evaluate the safety of ePIPAC treatment for patients with unresectable peritoneal metastasis as well as their tolerance and response to this treatment.

\section{METHODS}

\section{Study Design and Patient Selection}

This retrospective cohort study included all consecutive ePIPAC procedures performed at Dupuytren University Hospital (Limoges, France), Cancer Institute of Montpellier (Montpellier, France) and at Lausanne University Hospital (CHUV, Switzerland) between April 2019 and April 2020. All data were retrieved from prospective institutional databases. Indications were validated by the multidisciplinary tumor boards of each institution. Retrospective analysis was approved by the institutional review board in Lausanne (CER-VD 2019-00747), and the study was conducted in line with the declaration of Helsinki.

All the patients had unresectable PM without the curative option, and the exclusion criteria were according to previously published recommendations. ${ }^{6}$ The ePIPAC procedure was performed either alone or in association with systemic chemotherapy (one cycle ePIPAC and two cycles of systemic chemotherapy). ${ }^{10,11}$ The drug choice was based on the type of primary tumor, the preexistent response to oxaliplatin for digestive cancers, and the presence of neuropathy, as detailed elsewhere. ${ }^{6,12}$

The planned regimen included a standard of three procedures, but PIPAC treatment could be continued thereafter. When no clinical improvement was observed or early tumoral progression was identified, treatment was stopped prematurely after one or two ePIPACs.

\section{Surgical Procedure}

The PIPAC procedure was performed as previously described, ${ }^{13}$ with add-ons specific to electrostatic precipitation. An atraumatic stainless steel brush electrode was inserted through the abdominal wall using the Seldinger technique under direct visualization in accessible spot of the abdominal wall, but without the tip directly contacting any tissue (Fig. 1). A generator unit (Ultravision system, $7500-9500 \mathrm{~V}$, current $\leq 10 \mu \mathrm{A}$ ) was activated to produce the electrostatic attraction of the negatively charged aerosol particles to the peritoneal metastasis and the peritoneal tissue surface (reference electrode). The generator was activated at the surgeon's discretion either before or after the aerosolization (no accepted consensus).

Electrostatic precipitation is based on an add-on to the conventional PIPAC technique, as previously described in several experimental studies. An Ion Wand connected to the Ultravision system forms a dense electron cloud in front of the ioniser, which disperses in all directions in abdominal cavity, causing the aerosol of chemotherapy to be negatively charged and thus strongly attracted to the peritoneum, which is the nearest positively charged surface. ${ }^{9}$

\section{Outcomes}

The following data were recorded: demographic, clinical, operative and pathologic variables. For each ePIPAC, the Peritoneal Cancer Index (PCI) and volume of ascites were evaluated. The complete and major histologic responses (peritoneal regression grading score 1 [PRGS1] and PRGS2, respectively) were evaluated according to the previously reported classification. ${ }^{4}$ The mean PRGS per biopsy samples (at least 3) was used. Postoperative complications by procedure were classified according to the Clavien classification ${ }^{14}$ and toxicities by patient according to the Common Terminology Criteria for Adverse Events (CTCAE) v4.0. ${ }^{15}$

To assess quality of life (QoL) for the patients with unresectable peritoneal metastasis, the EORTC QLQ-C30 questionnaire ${ }^{16}$ was completed before each ePIPAC. Oncologic outcomes were analysed for patients undergoing repeated ePIPAC treatment, comparing PCI, ascites volume, histologic response, and QoL and according to the number of ePIPACs ( $<3$ vs $\geq 3$ ePIPACs).

\section{Statistical Analysis}

Continuous variables are presented as mean \pm standard deviation or as median (range) for skewed data. Categorical variables, compared using the Chi square test, are reported as frequencies (\%). A $p$ value of 0.05 was used to indicate 
FIG. 1 Photography of trocars and electrostatic electrode of the electrostatic precipitation pressurized intraperitoneal aerosol chemotherapy (ePIPAC) procedure

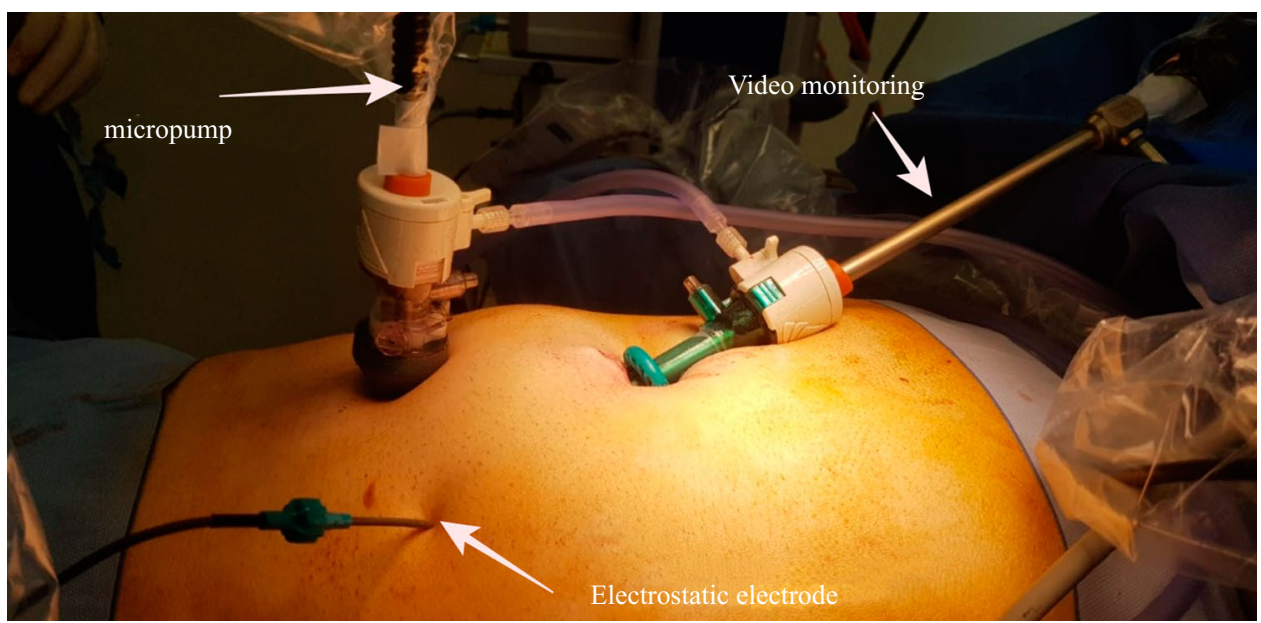

statistical significance. Efficacy was assessed for the patients with repeated PIPACs $(\geq 3)$ to avoid positive selection bias. Statistical analyses were performed and figures were produced with GraphPrism v.8.0 (GraphPad Software, San Diego, CA, USA).

\section{RESULTS}

\section{Characteristics of the Cohort}

In the three expert centers, 69 patients underwent 147 ePIPACs for nonresectable PMs (ratio, 2.1 ePIPACs per patient). The demographic characteristics of the cohort are presented in Table 1.

Of the 69 patients, 3 were treated with ePIPACs in the first line $(4.3 \%)$, and 66 had already been treated with at least one regimen of chemotherapy before ePIPAC comprising a median of eight cycles (range 1-40). More than two lines of preoperative chemotherapy were administered to 45 patients $(65.2 \%)$. The flowchart of the treatment is presented in Fig. 2.

\section{ePIPAC Procedure}

A total of 147 ePIPACs were performed, and 112 of the ePIPAC procedures $(76.2 \%)$ were alternated with systemic chemotherapy. The electrostatic generator was activated after chemotherapy aerosolization in 118 ePIPACs $(80.3 \%)$, and the median exposure time after activation was $12 \mathrm{~min}$ (range 6-30 min). The ePIPAC procedures are detailed in Table 2.

Three technical incidents were reported during the ePIPAC procedure (2\%): two automatic safety stops during the chemotherapy nebulisation when the pressure line reached the upper pressure limit and one contained leak of the high-pressure line.
Postoperative Complications and Treatment Toxicity in Both Groups

No hospital mortality among the 69 patients occurred during the 147 ePIPACs procedures. Of the 69 patients, overall and major toxicities according to CTCAE version 4.0 occurred at 30 days for respectively 17 patients $(24.6 \%)$ and 11 patients $(15.9 \%)$, as detailed in Table 3.

Among 147 ePIPACs, postoperative complications according to Clavien classification occurred at 30 days in four ePIPACs $(2.7 \%)$, and one serious complication (grade 4; $0.7 \%$ gaz embolism after open laparoscopy) was recorded (Table 3).

\section{Response to Treatment After the Third ePIPAC}

Three procedures or more could be performed for $45.7 \%$ of the patients in the cisplatin-doxorubicin group versus $19.7 \%$ of those in the oxaplatin group $(p=0.03)$. The comparison between the first and third treatments showed that both the PCI score ( 16 vs $14 ; p=0.4$ ) and the volume of ascites ( 320 vs $98 \mathrm{ml} ; p=0.1$ ) were stable during the treatment period (Fig. 3). For 22 patients who underwent three ePIPACs, the complete and major histologic responses were respectively $38.5 \%$ and $53.8 \%$. The mean PRGS was $2.2 \pm 1.1$ during the first ePIPAC and $1,7 \pm 0.6$ during the third ePIPAC $(p=0.15)$.

Response to Treatment in Both Groups $(<3$ vs $\geq 3$ ePIPACs)

After the third ePIPAC, an improvement in mean PRGS was found in seven patients (53.8\%), whereas a stable score was observed in two patients $(15.4 \%)$ and a worse score in four patients $(30.8 \%)$ (Table 4$)$. The global QoL score was stable up to the third ePIPAC and increased 
TABLE 1 Characteristics of 69 patients treated by ePIPAC

\begin{tabular}{|c|c|c|}
\hline Patient characteristics & No. or mean & $\%$ or $(\min -\max )$ \\
\hline Total patients & 69 & 100 \\
\hline \multicolumn{3}{|l|}{ Sex } \\
\hline Female & 34 & 49.3 \\
\hline Male & 35 & 50.7 \\
\hline Mean age: years (range) & 60 & $(33-88)$ \\
\hline Mean BMI: kg/m² (range) & 24 & $(16-36)$ \\
\hline ASA & 2.4 & \pm 0.48 \\
\hline \multicolumn{3}{|l|}{ Tumor type } \\
\hline Colorectal & 25 & 36.2 \\
\hline Ovarian & 14 & 20.3 \\
\hline Gastric & 13 & 18.8 \\
\hline Pancreatic & 5 & 7.2 \\
\hline Peritoneal mesothelioma & 4 & 5.8 \\
\hline Cholangiocarcinoma & 4 & 5.8 \\
\hline Small bowel & 1 & 1.4 \\
\hline Esophagus & 1 & 1.4 \\
\hline CUP & 1 & 1.4 \\
\hline Lung & 1 & 1.4 \\
\hline \multicolumn{3}{|l|}{ Previous systemic chemotherapy } \\
\hline First line & 3 & 4.3 \\
\hline Second line & 21 & 30.4 \\
\hline Third line & 33 & 47.8 \\
\hline Fourth line & 5 & 7.2 \\
\hline More than fourth line & 7 & 10.1 \\
\hline Mean cycle of chemotherapy (range) & 8 & $(0-40)$ \\
\hline \multicolumn{3}{|l|}{ Symptoms before the first ePIPAC } \\
\hline Nausea & 2 & 2.9 \\
\hline Abdominal pain & 10 & 14.5 \\
\hline Digestive disorders & 6 & 8.7 \\
\hline Asthenia & 5 & 7.2 \\
\hline \multicolumn{3}{|c|}{ Radiologic exams before the first ePIPAC $(n=69)$} \\
\hline CT scan & 65 & 94.2 \\
\hline Abdominal MRI & 10 & 14.5 \\
\hline PET scan & 13 & 18.8 \\
\hline
\end{tabular}

ePIPAC, electrostatic precipitation pressurized intraperitoneal aerosol chemotherapy; BMI, body mass index; ASA, American Society of Anesthesiologist; CUP, carcinoma of unknown primary origin; CT, computed tomography; MRI, magnetic resonance imaging; PET, positron emission tomography after the fourth ePIPAC procedure (56.5 vs $75 ; p=0.0002$; Fig. 4).

Four patients underwent complete cytoreduction and hyperthermic intraperitoneal chemotherapy (HIPEC) due to a peritoneal response after one $\operatorname{ePIPAC}(n=2$, colorectal PM) and a PM deemed resectable after three ePIPACs ( $n=2$, gastric PM). The median PCI was 5 at the time of the cytoreductive surgery (CRS) and HIPEC.

\section{DISCUSSION}

The current study confirmed the feasibility and safety of ePIPAC for patients who could be treated repeatedly for unresectable peritoneal metastasis. The principle of the ePIPAC technique is attractive for clinical practice because of its potential to enhance distribution and penetration of the chemotherapy in the PM. ${ }^{8,9}$ A recent study comparing PIPAC, ePIPAC with exposure of $1 \mathrm{~min}\left(\mathrm{ePIPAC}_{1 \mathrm{~min}}\right)$, and ePIPAC with exposure of $30 \mathrm{~min}\left(\mathrm{ePIPAC}_{30 \mathrm{~min}}\right)$ demonstrated the superiority of this technique over traditional 


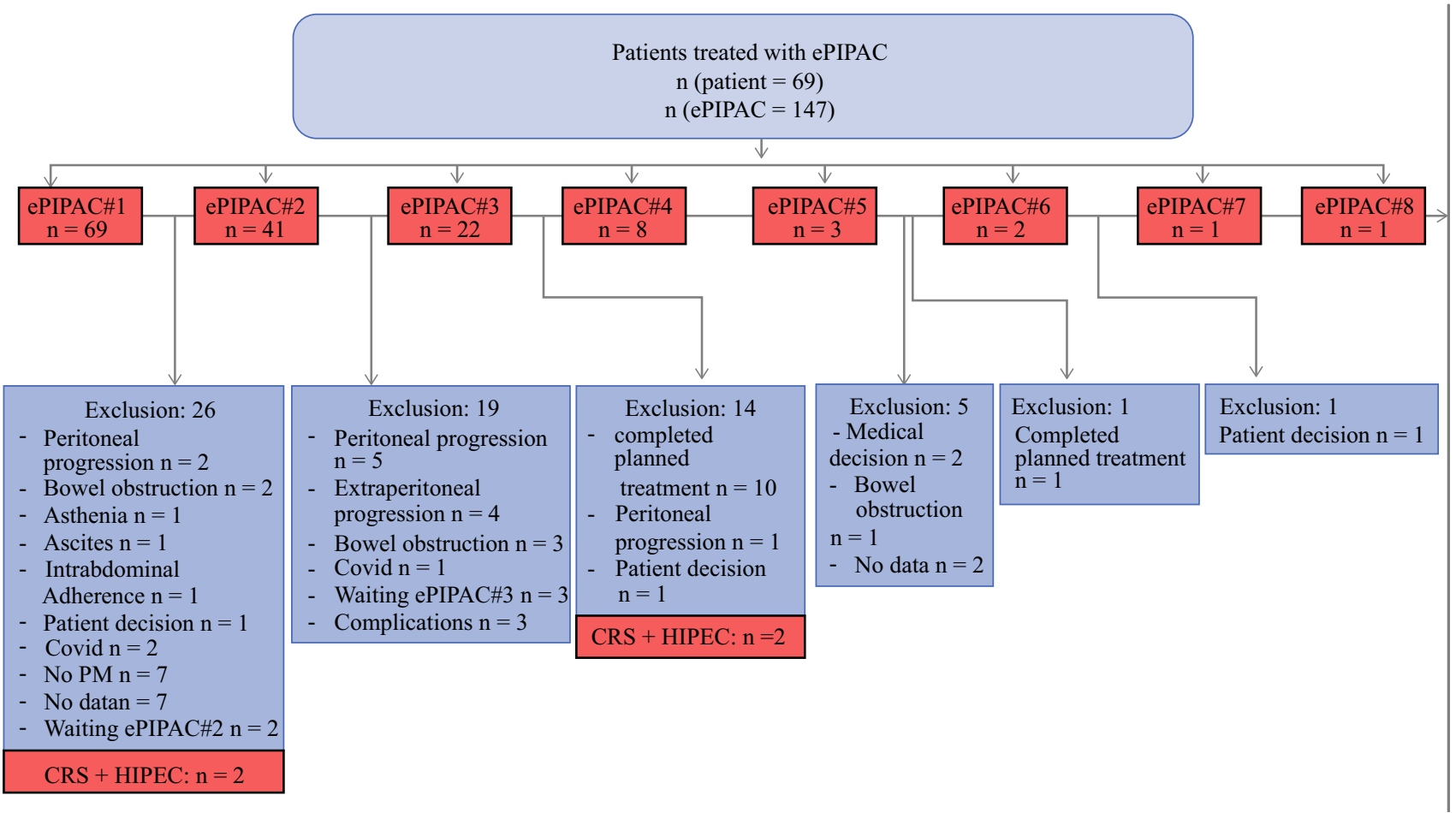

FIG. 2 Flowchart of patients during electrostatic precipitation pressurized intraperitoneal aerosol chemotherapy (ePIPAC) cycles

PIPAC in both an experimental model and an in vivo model with rats. ${ }^{17}$ The ePIPAC technique improved spatial distribution in both models and tissue penetration in the in vivo model based on nebulization of a nanoparticle (nabAbraxane). For specific positions related to the nozzle in the experimental model, the distribution was enhanced for ePIPAC $_{30 \text { min }}$ compared with ePIPAC ${ }_{1 \text { min }}$. No intermediary intervals were considered in this study.

The patients in our study were treated in centers that were early adopters of ePIPAC based on the experimental attractivity of this add-on, the initial safety reports, ${ }^{9}$ and the previously reported but unpublished results of the inventor group for an exposure time of 6 min. ${ }^{18}$ Indeed, the major CTCAE 4.0 toxicity was low (15.9\% of the patients), and the postoperative complications according to the Clavien classification were scarce (2.7\% of procedures). Most of the patients' symptoms were abdominal pain ascites, nausea, and asthenia, and the rates were comparable with those of other studies, ${ }^{6}$ and thus were probably more related to the intraperitoneal aerosolization than to the electrostatic add-on. Furthermore, in this cohort, ePIPAC was associated with systemic chemotherapy for the majority of the patients $(76 \%)$ without any additional toxicities, confirming the observation of another similar report. ${ }^{7}$

The median exposure time for these patients was $12 \mathrm{~min}$, as the choice was based on available evidence and evolved with the publication of new data. ${ }^{7,19}$ This allowed for a median reduction of the mean operating time of
24 min compared with the local experience of each center with conventional PIPAC. If intermediary exposure times are further validated for ePIPAC, then the electrostatic precipitation will be associated with cost-related benefits due to a significant reduction in the operating room time. A shorter time in the operating room probably implies fewer occupational safety hazards during the administration of the IP chemotherapy. ${ }^{20,21}$ Currently, however, a shortcourse PIPAC $_{1 \min }$ failed to prove clinical noninferiority compared with conventional PIPAC ${ }^{19}$ or experimental noninferiority compared with ePIPAC ${ }_{30 \text { min. }} \cdot{ }^{17}$

Other technical details of ePIPAC procedures are currently under investigation. The timing of the electrostatic device activation, which seems essential for ePIPAC, is empirically determined, with some centers favoring activation at the start of the cytotoxic administration and others favoring activation at the end of the administration time. ${ }^{22}$ In this multicentric study, the device was activated at the end of the administration time in $80 \%$ of cases, but to date no comparative translational evidence is available. The heterogeneity of practice was emphasized by a recent survey based on the responses from $93 \%$ of all identified PIPAC centers worldwide. ${ }^{18}$ The nine centers performing ePIPAC at the time of the survey reported a large diversity of preferences in time-related and generator activation-related items. Consequently, a Delphi consensus process was 
TABLE 2 ePIPAC procedures

\begin{tabular}{|c|c|c|c|c|c|c|}
\hline & \multicolumn{2}{|c|}{ ePIPAC $<3$} & \multicolumn{2}{|c|}{ ePIPAC $\geq 3$} & \multicolumn{2}{|l|}{ Total } \\
\hline & $n$ & $\%$ or $\min -\max$ & $n$ & $\%$ or $\min -\max$ & $n$ & $\%$ or $\min -\max$ \\
\hline No. of ePIPACs & 110 & 100 & 37 & 100 & 147 & 100 \\
\hline No. ePIPACs alone & 26 & 23.6 & 9 & 24.3 & 35 & 23.8 \\
\hline No. ePIPACs associated with systemic chemotherapy & 84 & 76.4 & 28 & 75.7 & 112 & 76.2 \\
\hline Mean delay between ePIPAC and systemic chemotherapy (weeks) & 3.9 & $(0-6)$ & 4 & $(0-6)$ & 3.92 & $(0-6)$ \\
\hline \multicolumn{7}{|l|}{ Type of IP chemotherapy } \\
\hline Oxaliplatin $\left(90 \mathrm{mg} / \mathrm{m}^{2}\right)$ & 2 & 1.8 & 0 & 0 & 2 & 1.4 \\
\hline Oxaliplatin $\left(92 \mathrm{mg} / \mathrm{m}^{2}\right)$ & 49 & 44.5 & 10 & 27.0 & 59 & 40.1 \\
\hline Cisplatin $\left(7.5 \mathrm{mg} / \mathrm{m}^{2}\right)$-doxorubicin $\left(1.5 \mathrm{mg} / \mathrm{m}^{2}\right)$ & 25 & 22.7 & 13 & 35.1 & 38 & 25.9 \\
\hline Cisplatin $\left(10.5 \mathrm{mg} / \mathrm{m}^{2}\right)$-doxorubicin $\left(2.1 \mathrm{mg} / \mathrm{m}^{2}\right)$ & 34 & 30.9 & 14 & 37.8 & 48 & 32.7 \\
\hline \multicolumn{7}{|l|}{ Duration of chemotherapy nebulization (min) } \\
\hline 5 & 56 & 50.9 & 12 & 32.4 & 68 & 46.3 \\
\hline 6 & 31 & 28.2 & 12 & 32.4 & 43 & 29.3 \\
\hline 8 & 23 & 20.9 & 13 & 35.1 & 36 & 24.5 \\
\hline \multicolumn{7}{|l|}{ Activation of electrostatic monitor } \\
\hline Before chemotherapy nebulization & 20 & 18.2 & 9 & 24.3 & 29 & 19.7 \\
\hline After chemotherapy nebulization & 90 & 81.8 & 28 & 75.7 & 118 & 80.3 \\
\hline \multicolumn{7}{|l|}{ Delay of electrostatic therapy } \\
\hline Minutes reported & 1 & 0.9 & 0 & 0 & 1 & 0.7 \\
\hline 6 & 64 & 58.2 & 19 & 51.4 & 83 & 56.5 \\
\hline 10 & 4 & 3.6 & 1 & 2.7 & 5 & 3.4 \\
\hline 12 & 6 & 5.5 & 3 & 8.1 & 9 & 6.1 \\
\hline 15 & 14 & 12.7 & 10 & 27.0 & 24 & 16.3 \\
\hline 20 & 1 & 0.9 & 0 & 0 & 1 & 0.7 \\
\hline 30 & 20 & 18.2 & 4 & 10.8 & 24 & 16.3 \\
\hline
\end{tabular}

ePIPAC, electrostatic precipitation pressurized intraperitoneal aerosol chemotherapy

launched to harmonize practice of PIPAC, including details on electrostatic precipitation (timing of activation and exposure time).

The response evaluation after this procedure is as important as safety in validating the use of ePIPAC in clinical practice. In the current study, response was based on both clinical and histologic criteria. Clinical parameters (PCI and volume of ascites) were stable in a comparison between the first and third procedures. In the study, PRGS was proposed but not completely validated as a surrogate parameter for efficacy in the PIPAC treatment. Despite PRGS stability between the first and third procedures in the current cohort, the time of exposure to chemotherapy during ePIPAC may be important to production of the histologic effect, as confirmed recently by Graversen et al. ${ }^{19}$

Due to the limited number of patients and the absence of a comparative arm with conventional PIPAC, nothing can be inferred about the supplementary efficacy of ePIPAC versus PIPAC. This study may show that even with a shorter procedure, patients with PMs have at least stable disease during the treatment period. With the palliative strategy, many authors have suggested that stable disease after oncologic treatment might confirm its efficacy and even more so in additional lines of treatment. $^{23,24}$ This palliative therapy aims to treat the peritoneal metastasis directly and to stabilize or decrease the tumor proliferation while at the same time not altering the QoL of the patients, as does standard PIPAC. ${ }^{25}$ Although, we were limited by the number of questionnaires returned by the patients and the patients who stopped the ePIPAC therapy, the QoL was stable up to the third ePIPAC treatment and improved significantly for the patients who could receive more than three ePIPACs.

The main limitations of this study were related to the small and heterogeneous cohort of patients presenting with unresectable PM of different origins, to the diversity of practices related to exposure time and activation of the generator, to the absence of radiologic correlations, and to the short follow-up period. Although, the study had the 
TABLE 3 Postoperative outcomes after ePIPAC

\begin{tabular}{|c|c|c|c|c|c|c|}
\hline & \multicolumn{2}{|c|}{ ePIPAC $<3$} & \multicolumn{2}{|c|}{ ePIPAC $\geq 3$} & \multicolumn{2}{|c|}{ Total } \\
\hline & $n$ & $\%$ & $n$ & $\%$ & $n$ & $\%$ \\
\hline No. of ePIPACs & 110 & 100 & 37 & 100 & 147 & 100 \\
\hline Intraoperative complications & 0 & 0 & 0 & 0 & 0 & 0 \\
\hline ePIPAC incidents & 2 & 1.8 & 1 & 2.7 & 3 & 2.0 \\
\hline \multicolumn{7}{|l|}{ Postoperative complications } \\
\hline Toxicities (CTCAE grade) & 16 & 14.5 & 0 & 0 & 16 & 10.8 \\
\hline Nausea (2) & 2 & 1.8 & 0 & 0 & 2 & 1.4 \\
\hline Acute urinary retention (2) & 2 & 1.8 & 0 & 0 & 2 & 1.4 \\
\hline Asthenia (2) & 2 & 1.8 & 0 & 0 & 2 & 1.4 \\
\hline Pain (3) & 5 & 4.5 & 0 & 0 & 5 & 3.4 \\
\hline Postoperative occlusion (3) & 2 & 1.8 & 0 & 0 & 2 & 1.4 \\
\hline Ascites (3) & 3 & 2.7 & 0 & 0 & 3 & 2 \\
\hline Anaphylactic shock (4) & 0 & 0 & 1 & 2.7 & 1 & 0.7 \\
\hline Postoperative complications (Clavien grade) & 4 & 3.6 & 0 & 0 & 4 & 2.7 \\
\hline Subcutaneous hematoma (2) & 2 & 1.8 & 0 & 0 & 2 & 1.4 \\
\hline Cholecystisis (3) & 1 & 0.9 & 0 & 0 & 1 & 0.7 \\
\hline Gas embolism (4) & 1 & 0.9 & 0 & 0 & 1 & 0.7 \\
\hline
\end{tabular}

ePIPAC, electrostatic precipitation pressurized intraperitoneal aerosol chemotherapy; CTCAE, Common Terminology Criteria for Adverse Events
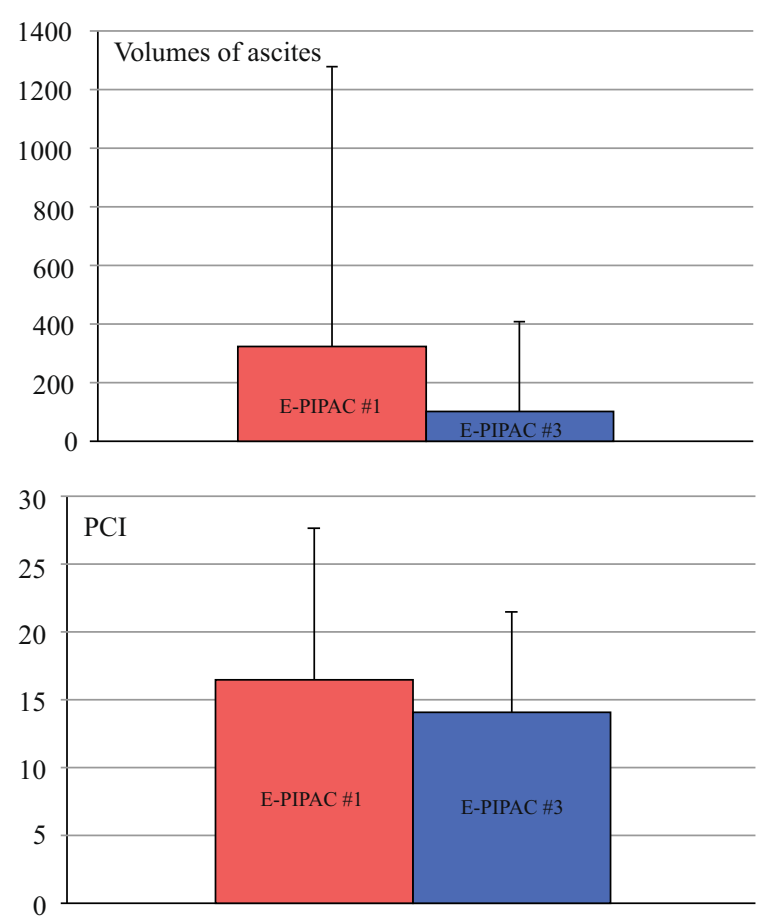

FIG. 3 Response to treatment 1 month after the third cycle of electrostatic precipitation pressurized intraperitoneal aerosol chemotherapy (ePIPAC): histologic response (peritoneal regression

largest cohort of patients analyzed to date, the procedural details varied considerably, and a large number of patients could not undergo the full course of at least three treatments. Nevertheless, in the current development stage of
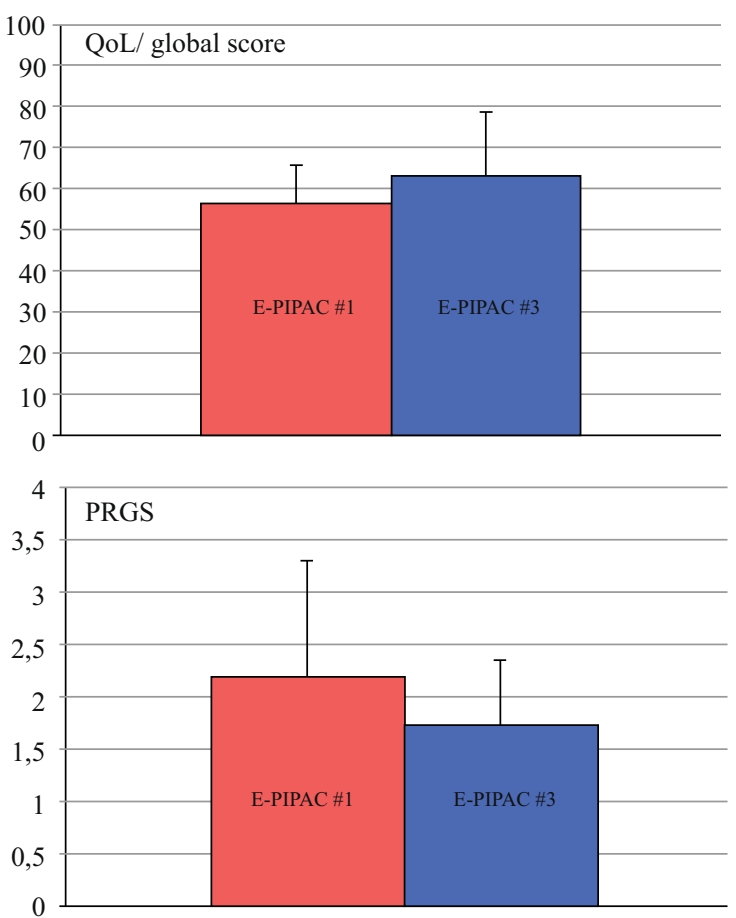

grade score [PRGS]), Peritoneal Cancer Index (PCI), volume of ascites, and quality of life were stable between ePIPACs 1 and 3

ePIPAC, these aspects are inherent to any multicentric cohort, whereas preliminary clinical results are needed to stimulate the creation of future prospective trials. 
TABLE 4 Peritoneal Cancer Index (PCI), volume of ascites, and quality of life (EORTC-QLQ-C30) after repetitive ePIPAC

\begin{tabular}{|c|c|c|c|c|c|c|c|}
\hline & \multicolumn{2}{|l|}{ ePIPAC $<3$} & \multicolumn{2}{|l|}{ ePIPAC $\geq 3$} & \multirow[t]{2}{*}{$p$ value } & \multicolumn{2}{|l|}{ Total } \\
\hline & $N$ & $\%$ or $\min -\max$ & $N$ & $\%$ or $\min -\max$ & & $n$ & $\%$ or $\min -\max$ \\
\hline No. of ePIPACs & 110 & 100 & 37 & 100 & & 147 & 100 \\
\hline EORTC-QLQ-C30 & $59.1(n=18)$ & $(48-83)$ & $68.4(n=15)$ & $(41-83)$ & 0.05 & $66.5(n=33)$ & $(41-83)$ \\
\hline Ascites (ml) & 364 & $(0-8000)$ & 252 & $(0-1500)$ & NS & 267 & $(0-8000)$ \\
\hline PCI score & 16.3 & $(0-39)$ & 15 & $(1-31)$ & NS & 16 & $(0-39)$ \\
\hline
\end{tabular}

ePIPAC, electrostatic precipitation pressurized intraperitoneal aerosol chemotherapy; NS, not significant; PCI, Peritoneal Cancer Index

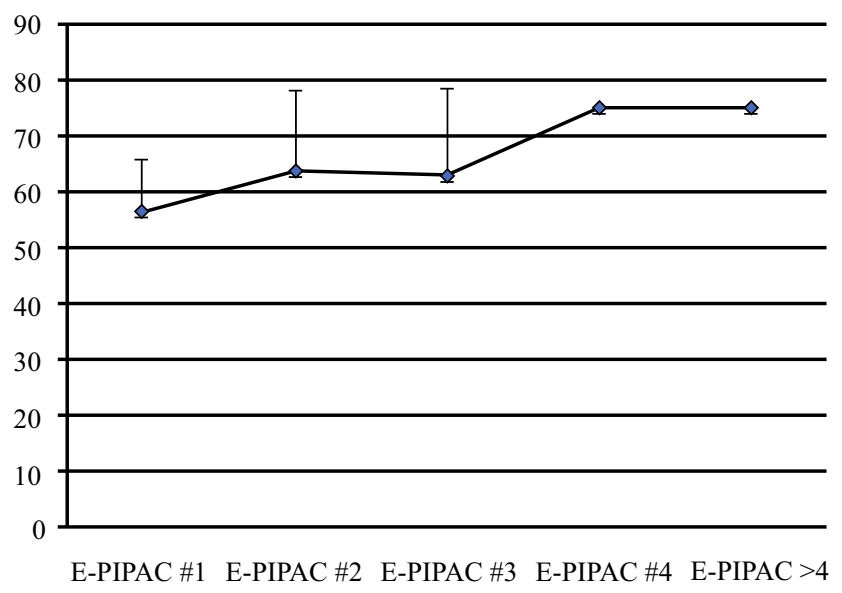

FIG. 4 Quality of life during ePIPAC cycles

In conclusion, repeated ePIPAC alone or ePIPAC associated with systemic chemotherapy was feasible, safe, and well-tolerated. Efficacy of ePIPAC and standard PIPAC must be further studied in the multicenter setting by tumor entity. The short-term to do list includes the urgently needed standardization of technical details (ongoing Delphi consensus) and an improved selection of patients for PIPAC treatment.

DISCLOSURE There are no conflicts of interest.

\section{REFERENCES}

1. Girshally R, Demtröder C, Albayrak N, Zieren J, Tempfer C, Reymond MA. Pressurized intraperitoneal aerosol chemotherapy (PIPAC) as a neoadjuvant therapy before cytoreductive surgery and hyperthermic intraperitoneal chemotherapy. World J Surg Oncol. 2016;14:253.

2. Robella M, Vaira M, De Simone M. Safety and feasibility of pressurized intraperitoneal aerosol chemotherapy (PIPAC) associated with systemic chemotherapy: an innovative approach to treat peritoneal carcinomatosis. World $J$ Surg Oncol. 2016;14:128.

3. Solass W, Giger-Pabst U, Zieren J, Reymond MA. Pressurized intraperitoneal aerosol chemotherapy (PIPAC): occupational health and safety aspects. Ann Surg Oncol. 2013;20:3504-11.
4. Solass W, Sempoux C, Detlefsen S, Carr NJ, Bibeau F. Peritoneal sampling and histological assessment of therapeutic response in peritoneal metastasis: proposal of the Peritoneal Regression Grading Score (PRGS). Pleura Peritoneum. 2016;1(2):99-107.

5. Benzerdjeb N, Durieux E, Tantot J, Isaac S, Fontaine J, Harou O, et al. Prognostic impact of combined progression index based on peritoneal grading regression score and peritoneal cytology in peritoneal metastasis. Histopathology. 2020.

6. Alyami M, Hübner M, Grass F, Bakrin N, Villeneuve L, Laplace $\mathrm{N}$, et al. Pressurised intraperitoneal aerosol chemotherapy: rationale, evidence, and potential indications. Lancet Oncol. 2019;20:e368-77.

7. Willaert W, Van de Sande L, Van Daele E, Van De Putte D, Van Nieuwenhove Y, Pattyn P, et al. Safety and preliminary efficacy of electrostatic precipitation during pressurized intraperitoneal aerosol chemotherapy (PIPAC) for unresectable carcinomatosis. Eur J Surg Oncol. 2019;45:2302-9.

8. Kakchekeeva T, Demtröder C, Herath NI, Griffiths D, Torkington $\mathrm{J}$, Solaß W, et al. In vivo feasibility of electrostatic precipitation as an adjunct to pressurized intraperitoneal aerosol chemotherapy (ePIPAC). Ann Surg Oncol. 2016;23(Suppl 5):592-8.

9. Reymond M, Demtroeder C, Solass W, Winnekendonk G, Tempfer C. Electrostatic precipitation pressurized intraperitoneal aerosol chemotherapy (ePIPAC): first in-human application. Pleura Peritoneum. 2016;1:109-16.

10. Alyami M, Mercier F, Siebert M, Bonnot P-E, Laplace N, Villeneuve $\mathrm{L}$, et al. Unresectable peritoneal metastasis treated by pressurized intraperitoneal aerosol chemotherapy (PIPAC) leading to cytoreductive surgery and hyperthermic intraperitoneal chemotherapy. Eur J Surg Oncol. 2019;S0748-7983(19)30522-0.

11. Nowacki M, Alyami M, Villeneuve L, Mercier F, Hubner M, Willaert W, et al. Multicenter comprehensive methodological and technical analysis of 832 pressurized intraperitoneal aerosol chemotherapy (PIPAC) interventions performed in 349 patients for peritoneal carcinomatosis treatment: an international survey study. Eur J Surg Oncol. 2018;44:991-6.

12. Nagata $T$, Fukuda K-I, Tamai M, Taniguchi A, Kamiya H, Kambe K, et al. Early neuropathy related to oxaliplatin treatment in advanced and recurrent colorectal cancer. Anticancer Res. 2019;39:1347-53.

13. Sgarbura O, Hübner M, Alyami M, Eveno C, Gagnière J, Pache $\mathrm{B}$, et al. Oxaliplatin use in pressurized intraperitoneal aerosol chemotherapy (PIPAC) is safe and effective: a multicenter study. Eur J Surg Oncol. 2019;45:2386-91.

14. Clavien PA, Barkun J, de Oliveira ML, Vauthey JN, Dindo D, Schulick RD, et al. The Clavien-Dindo classification of surgical complications: five-year experience. Ann Surg. 2009;250:187-96.

15. National Cancer Institute. Common Terminology Criteria for Adverse Events (CTCAE), version 4.0. Bethesda, MD: National Cancer Institute; 2009. 
16. van der Kloot WA, Kobayashi K, Yamaoka K, Inoue K, Nortier HWR, Kaptein AA. Summarizing the fifteen scales of the EORTC QLQ-C30 questionnaire by five aggregate scales with two underlying dimensions: a literature review and an empirical study. J Psychosoc Oncol. 2014;32:413-30.

17. Van de Sande L, Willaert W, Cosyns S, De Clercq K, Shariati M, Remaut K, et al. Establishment of a rat ovarian peritoneal metastasis model to study pressurized intraperitoneal aerosol chemotherapy (PIPAC). BMC Cancer. 2019;19:424.

18. Sgarbura $\mathrm{O}$, et al, Current practice of pressurized intraperitoneal aerosol chemotherapy (PIPAC): still standardized or on the verge of diversification? Eur J Surg Oncol. 2020;S0748-7983(20) 30712-5. https://doi.org/10.1016/j.ejso.2020.08.020.

19. Graversen M, Detlefsen S, Ellebaek SB, Fristrup C, Pfeiffer P, Mortensen MB. Pressurized intraperitoneal aerosol chemotherapy with one minute of electrostatic precipitation (ePIPAC) is feasible, but the histological tumor response in peritoneal metastasis is insufficient. Eur J Surg Oncol. 2020;46:155-9.

20. Ametsbichler P, Böhlandt A, Nowak D, Schierl R. Occupational exposure to cisplatin/oxaliplatin during pressurized intraperitoneal aerosol chemotherapy (PIPAC)? Eur J Surg Oncol. 2018;44:1793-9.

21. Ndaw S, Hanser O, Kenepekian V, Vidal M, Melczer M, Remy $\mathrm{A}$, et al. Occupational exposure to platinum drugs during intraperitoneal chemotherapy: biomonitoring and surface contamination. Toxicol Lett. 2018;298:171-6.

22. Sautkin I. In: 5th PIPAC symposium, 7-8 Sept 2018, Paris. https://sites.altilab.com/files/CONGRES/2018/PIPAC2018.pdf.

23. Popov I, Jelić S, Radosavljević D, Nikolić-Tomasević Z. The role of stable disease in objective response assessment and its impact on survival in advanced colorectal cancer: is "stable disease" a homogenous response category? Neoplasma. 1999;46:132-9.

24. Tamura T, Kurishima K, Nakazawa K, Ishikawa H, Satoh H, Hizawa N. Similar survival benefits of a good response and stable disease to platinum-based chemotherapy in non-small cell lung cancer. Oncol Lett. 2015;10:1135-40.

25. Odendahl K, Solass W, Demtröder C, Giger-Pabst U, Zieren J, Tempfer C, et al. Quality of life of patients with end-stage peritoneal metastasis treated with pressurized intraperitoneal aerosol chemotherapy (PIPAC). Eur $J$ Surg Oncol. 2015;41:1379-85.

Publisher's Note Springer Nature remains neutral with regard to jurisdictional claims in published maps and institutional affiliations. 